\title{
Prevalence of Metabolic Syndrome among Students of Faculty of Health Science and Technology in Ebonyi State University, Abakaliki, Nigeria
}

\author{
Anya Kingsley K. ${ }^{1}$; Okoro Godwin O. ${ }^{1}$; Onyenekwe Charles C. ${ }^{1}$; Fasogbon Samuel Ayobami ${ }^{*}$; Adebayo \\ Ahmed O. ${ }^{3}$ \\ ${ }^{1}$ Department of Medical Laboratory Science, Ebonyi State University \\ Abakaliki, Ebonyi state, Nigeria \\ ${ }^{2}$ Public Health In-vitro Diagnostic Control Laboratory, Medical Laboratory Science Council of Nigeria (MLSCN) \\ Yaba-Lagos, Nigeria \\ ${ }^{3}$ Department of Medical Laboratory Science, Lagos State College of Health Technology \\ Yaba-Lagos, Nigeria \\ *Corresponding author's email: samfash4best [AT] gmail.com
}

\begin{abstract}
Metabolic impairments could be seen at any point in human development. Although emphasis has been placed on older adults but it could be encountered in any age brackets. The aim of this study was to compare prevalence rates using different definitions of Metabolic syndrome (MetS) among students of Faculty of Health Sciences and Technology (FHST) in Ebonyi State University (EBSU), Abakaliki. This was a cross-sectional study involving 80 students (28 male students and 52 female students) recruited from among students of FHST in EBSU, Abakaliki, Ebonyi State, Nigeria. MetS was defined in three different ways [by International Diabetes Federation (IDF), National Cholesterol Education Program-Adult Treatment Panel III (NCEP-ATPIII), or World Health Organization (WHO) criteria]. Prevalence was found to be 6.3\%, $2.5 \%$ and $7.5 \%$ using NCEP-ATP III, WHO, and IDF definitions respectively. The most common MetS components among female students using the NCEP-ATP III criteria were high blood pressure (5.8\%) and abdominal obesity (5.8\%), whilst low HDL-C concentrations, high blood pressure and abdominal obesity were most common among male students $(7.1 \%$ for each component mentioned). According to WHO, abdominal obesity (7.1\%) and hyperglycaemia (7.1\%) were the most common MetS components among male students. The most common MetS components among participants using the IDF criteria were abdominal obesity and high blood pressure. While some participants did not meet the MetS criteria of the NCEP-ATP III, WHO and IDF criteria (50\%), many had one (30\%) or two (13.8\%) components and may be at risk of developing the syndrome in the future. The mean values of the risk factors used as criteria for the diagnosis of MetS were relatively normal in the study population thereby masking the presence of MetS, thus showing that the prevalence of MetS may gradually increase undetected unless individual members of the study population are subjected to laboratory investigation using different criteria for diagnosis of MetS. Therefore, traditional risk factors might be late markers for diagnosis of MetS since findings from this study showed that MetS was only detected in participants that already had it.
\end{abstract}

Keywords - Prevalence, Metabolic Syndrome, Blood pressure, Obesity

\section{INTRODUCTION}

Metabolic syndrome (MetS) is a global health problem currently occupying the front burner in clinical and public health practice [1]. It is a cluster of biochemical and anthropometric abnormality that has high predictive ability for the development of atherosclerotic cardiovascular diseases and type two diabetes mellitus (T2DM) [2] and has been variously defined with sets of convergent and divergent diagnostic criteria which vary in specific principal elements but generally include hypertension (HTN), obesity, glucose intolerance, hypertriglyceridaemia and reduced high density lipoprotein-cholesterol (HDL-C)-dyslipidaemia. [3]

Several studies have shown that the variability of MetS syndrome within and across the world populations manifest as a consequence of actions and interactions of genetic, [4] and non-genetic (socio-environmental) factors. The predominant underlying risk factors of MetS appear to be insulin resistance and abdominal obesity. The associated clinical conditions include physical inactivity, sedentary lifestyle [5,6] and other enhancing risk factors such as cigarette smoking and heavy alcohol consumption. 
Biological and socio-behavioural variations exist in the prevalence of metabolic syndrome (MetS). As the case detection of MetS increases in Nigeria, describing its prevalence and risk factors remain relevant for proactive control interventions.

Between 1998 and 2005, a number of definitions of the metabolic syndrome (MetS) have been proposed. This study was therefore set to compare prevalence rates of different definitions of MetS among students of faculty of health science and technology (FHST) in Ebonyi state university (EBSU), Abakaliki.

The prevalence of MetS has risen globally in the past 2 decades. [3,7] This trend is expected to continue predominantly in developing countries if no control interventions are put in place. [8] The prevalence of metabolic syndrome differs by definitions and diagnostic criteria across different parts of the world population. In United States of America the prevalence varies from $16 \%$ of black men to $37 \%$ of Hispanic women [5], prevalence of $39.3 \%$ was reported in Saudi Arabia [9], 29.7\% was reported in India, [10] 43.3\% was reported in Ethiopia,[11] 35.9\% was reported in Ghana,[12] 15.9\% was reported in South eastern Nigeria [13] and 14.9\% was reported in Abuja, North central Nigeria.[15] The prevalence of metabolic syndrome has also been reported in specific high risk patients such as those with T2DM, [4,15,16,17], hypertensive patients [18] and obese patients [19]. Among the patients with T2DM, the prevalence of metabolic syndrome of $77.2 \%$ was reported in diabetics in India. [20], 66.7\% was reported in diabetics in Eastern Nigeria, [17] 25.2\% was reported in diabetics in Western Nigeria [15] and 54.3\%\% was reported in Northern Nigeria. [16] In hypertensive patients' prevalence of $15.9 \%$ was reported in South-eastern Nigeria [13] and prevalence of $42.9 \%$ was reported in Western Nigeria. [21] Whilst in obese patients, prevalence of 46.3\% was reported in Qatar, [18] 40.2\% was reported in Malaysia [22] and 53.0\% was reported in Italy [19].

It is a known fact that chances of developing CVD in individuals with MetS is greatly increased when compared to the normal population independently of the diagnostic criteria used. Overall a range of 1.5-3.0 times greater risk of CVD and CHD mortality has been found in several prospective studies whereas a recent meta-analysis documented a twofold increase in cardiovascular outcomes and a 1.5-fold increase in all-cause mortality in individuals with MetS [23]. The ability of Mets to predict risk for T2DM has been extensively studied and it is well accepted that the presence of MetS not only increases this risk, but is also highly predictive of new-onset T2DM [24]. In fact, MetS is associated with an approximately five times higher risk for incident T2DM [6].

\section{MATERIALS AND METHODS}

\subsection{Study Population}

The study population was made up of 80 adult students aged between 18 years and above were screened for MetS using WHO, IDF and NCEP: ATP III criteria.

\subsection{Study Design and Setting}

This was a cross-sectional study conducted at college of health science (CHS) popularly known as Presco campus Abakaliki, during the months of July 2016 and August 2016. Study participants were current students of the faculty of health science and technology (FHST), Ebonyi State University (EBSU) in Abakaliki during the 2015-2016 academic sessions. Participants were selected based on their relatively high stability and willingness to participate in the study during the 2015-2016 academic sessions.

\subsection{Data Collection and Variable Specification}

The data collection exercise were carried in two phases; on day one, participant's physical/anthropometric measurements were taken and were asked to return the following day (day two) for the collection of fasting blood samples for the biochemical investigation during morning hours (7:30 a.m.-11.30. a.m.). The biochemical investigation were carried out on the day two of each data collection exercise after collection of fasting blood sample had ended with the assistance of three (3) medical laboratory scientists at the chemical pathology laboratory, phase 2 of federal teaching hospital (FETHA 2) Abakaliki, Ebonyi State. Blood samples were collected after a 12-hour overnight fasting. Samples of $7 \mathrm{~mL}$ of blood were obtained from each participant employing standard infection prevention procedures. These samples were collected with the aid of an experienced nurse. The collected aliquots of blood serum were used to determine participants' fasting glucose concentrations and lipid profiles. Serum triglycerides (TGs), total cholesterol (TC), highdensity lipoprotein cholesterol (HDL-C), low density lipoprotein cholesterol (LDL-C), and fasting serum glucose (FG) were measured with the assistance of three (3) medical laboratory scientists (using Erba Manheim Chem7 semi-auto analyzer spectrophotometer) at chemical pathology laboratory, phase 2 of federal teaching hospital (FETHA 2) Abakaliki, Ebonyi State. Lipid profile was determined by standardised enzymatic procedures using cholesterol assays. Participants' FG was determined using standardized glucose oxidase method. All laboratory assays were carried out using Randox reagents and test kits. Also all laboratory assays were completed without knowledge of participants' medical history. Lipid profile and FG concentrations were reported as mmol/l. 


\subsection{Sampling Technique}

A multistage, stratified sampling strategy was used to identify and recruit participants. Students were selected from the various classes/levels in the two departments (Department Of Medical Laboratory Science And Nursing Sciences) that made up the faculty. Students were approached during school hours and were invited to participate in the study, and the final sample size included 28 male and 52 female students giving a total of 80 participants.

\subsection{Physical/Anthropometric measurements}

Blood pressure (BP) was measured using a digital measuring device (Microlife BP A50, Microlife AG, Switzerland) with participants sitting after resting for at least five minutes. Three BP measurements were taken with at least three minute intervals between consecutive measurements. The mean systolic and diastolic BP from the second and third measurements was analysed [25]. The weights of the participants measured using a scale. Height and weight were measured with participants wearing light clothing. Waist circumference was taken at the midpoint between the lower margin of the last palpable rib and the top of the iliac crest (hip bone) [25]. Hip circumference was taken around the maximum circumference of the buttocks [25]. Body Mass Index (BMI) was used to measure overweight and obesity and was also used to determine abdominal obesity when BMI of participants exceeded $30 \mathrm{~kg} / \mathrm{m}^{2}$. BMI is calculated by the using the formula; $\frac{\text { Weight }}{\text { Height } 2}\left(\mathrm{~kg} / \mathrm{m}^{2}\right)$.

\subsection{Ethical Consideration and informed consent}

Ethical certificate was obtained from the Health Research and Ethics Committee of Ebonyi State University. Informed consent was also obtained from participants used in the study.

\subsection{Biochemical investigations}

\subsubsection{Estimation of Total Cholesterol (TC)}

Principle: The cholesterol is determined after the enzymatic hydrolysis and oxidation. The indicator quinoneimine is formed by hydrogen peroxide and 4-aminoantipyrine in the presence of phenol and peroxidase.

Procedure: Using a micropipette, $10 \mu \mathrm{l}$ of distilled water, $10 \mu \mathrm{l}$ of the standard and $10 \mu \mathrm{l}$ of the test sample was added to the test tubes designated (properly labelled test tubes) for the reagent blank, standard and test sample respectively. 1000 $\mu \mathrm{l}$ of reagent was added to each of the three (3) test tubes mentioned above. After which these test tubes were mixed respectively and incubated for 10 minutes at $20-25^{\circ} \mathrm{C}$.After incubation, small volumes of the reagent blank, standard and test sample were transferred into the cuvettes respectively.The concentration of the test sample was read against the reagent blank at $500 \mathrm{~nm}$ wavelength.

\subsubsection{Estimation of Triglycerides (TGs)}

\section{Method: Colorimetric method}

Principle: The triglycerides are determined after the enzymatic hydrolysis with lipase. The indicator quinoneimine is formed by hydrogen peroxide, 4-aminophenazone and 4-chlorophenol under the catalytic influence of peroxidase.

Procedure: Using a micropipette, $10 \mu \mathrm{l}$ of the standard and $10 \mu \mathrm{l}$ of the test sample were added to the test tubes designated for the standard and test sample respectively. $1000 \mu \mathrm{l}$ of the reagent were added each to the two (2) test tubes mentioned above and also to the test tube designated for the reagent blank. After which these test tubes were mixed respectively and incubated for 10 minutes at $20-25^{\circ} \mathrm{C}$.After incubation, small volumes of the reagent blank and test sample were transferred into the cuvettes respectively.The concentration of the test sample was read against the reagent blank at $500 \mathrm{~nm}$ wavelength.

\subsubsection{Estimation of HDL-Cholesterol (HDL)}

Principle: Low density lipoproteins (LDL and VLDL) and chylomicron fraction are precipitated out quantitatively by the addition of phosphotungstic acid in the presence of magnesium ions. After centrifugation, the cholesterol concentration in the HDL fraction, which remains in the supernatant, is determined.

Procedure: The procedure for analysing HDL-Cholesterol wasmade up of two stages- the first stage involved precipitation while the second stage did not.

Stage I-Precipitation: Using a micropipette, $200 \mu \mathrm{l}$ of the standard and $200 \mu 1$ of the test sample were added into the centrifuge tubes designated for the standard and test sample respectively.500 $\mu 1$ of diluted precipitant (RI) were added to the two (2) centrifuge tube mentioned above. After which these centrifuge tubes were mixed respectively and allowed to sit for 10 minutes at room temperature. Then the test sample and standard were centrifuged for 10 minutes at $3000 \mathrm{rpm}$. After centrifuging the clear supernatant was separated using a micropipette.

Stage II-Colorimetric: Using a micropipette, $100 \mu \mathrm{l}$ of distilled water, $100 \mu \mathrm{l}$ of the standard and $100 \mu \mathrm{l}$ of the supernatant were added to the test tubes designated (properly labelled test tubes) for the reagent blank, standard and test sample respectively. $1000 \mu 1$ of reagent was added to each of the three (3) test tubes mentioned above. After which these test tubes were mixed respectively and incubated for 10 minutes at $20-25^{\circ} \mathrm{C}$.After incubation, small volumes of the 
reagent blank, standard and test sample were transferred into the cuvettes respectively.The concentration of the test sample was read against the reagent blank at $500 \mathrm{~nm}$ wavelength.

\subsubsection{Estimation of Blood Glucose level}

\section{Glucose Oxidase Enzymatic Method}

Principle: Glucose is determined after enzymatic oxidation in the presence of glucose oxidase. The hydrogen peroxide formed reacts, under catalysis of peroxidase, with phenol and 4-aminophenazone to form red-violet quinoneimine.

Procedure: Using a micropipette, $10 \mu \mathrm{l}$ of the standard and $10 \mu \mathrm{l}$ of the test sample were added to the test tubes designated for the standard and test sample respectively. $1000 \mu 1$ of the reagent were added each to the two (2) test tubes mentioned above and also to the test tube designated for the reagent blank. After which these test tubes were mixed respectively and incubated for 10 minutes at $20-25^{\circ} \mathrm{C}$.After incubation, small volumes of the reagent blank, standard and test sample were transferred into the cuvettes respectively. The concentration of the test sample was read against the reagent blank at $500 \mathrm{~nm}$ wavelength.

\subsection{Diagnostic Criteria for Metabolic Syndrome Using WHO, NCEP: ATPIII AND IDF criteria}

Prevalence of MetS was defined according to WHO NCEP: ATPIII AND IDF criteria outlined below:

-World Health Organization criteria [26] Insulin resistance is defined as type 2 diabetes mellitus (DM) or impaired fasting glucose (IFG) (> $100 \mathrm{mg} / \mathrm{dl}$ or $5.6 \mathrm{mmol} / \mathrm{l}$ ) or impaired glucose tolerance (IGT), plus two of the following: (1) Abdominal obesity (waist-to-hip ratio $>0.9$ in men or $>0.85$ in women, or body mass index $(\mathrm{BMI})>30 \mathrm{~kg} / \mathrm{m}^{2} ;(2)$ Triglycerides $150 \mathrm{mg} / \mathrm{dl}(1.7 \mathrm{mmol} / \mathrm{l})$ or greater, and/or high-density lipoprotein (HDL)-cholesterol $<40 \mathrm{mg} / \mathrm{dl}(1.03$ $\mathrm{mmol} / \mathrm{l})$ in men and $<50 \mathrm{mg} / \mathrm{dl}(1.29 \mathrm{mmol} / \mathrm{l})$ in women; (3) Blood pressure (BP) 140/90 $\mathrm{mmHg}$ or greater ; (4) Microalbuminuria (urinary albumin secretion rate $20 \mu \mathrm{g} / \mathrm{min}$ or greater, or albumin-to-creatinine ratio $30 \mathrm{mg} / \mathrm{g} \mathrm{or}$ greater).

-National Cholesterol Education Program Adult Treatment Panel III (NCEP: ATPIII) criteria [NCEP: ATPIII 2001] Any three or more of the following: (1) Waist circumference $>102 \mathrm{~cm}$ in men, $>88 \mathrm{~cm}$ in women ; (2) Triglycerides 150 $\mathrm{mg} / \mathrm{dl}(1.7 \mathrm{mmol} / \mathrm{l})$ or greater ; (3) HDL-cholesterol < $40 \mathrm{mg} / \mathrm{dl}(1.03 \mathrm{mmol} / \mathrm{l})$ in $\mathrm{men}$ and < $50 \mathrm{mg} / \mathrm{dl}(1.29 \mathrm{mmol} / \mathrm{l}) \mathrm{in}$ women ; (4) BP 130/85 mmHg or greater ; (5) Fasting glucose $110 \mathrm{mg} / \mathrm{dl} *$ or greater.

* In 2003, the American Diabetes Association (ADA) changed the criteria for IFG tolerance from $110 \mathrm{mg} / \mathrm{dl}$ to 100 $\mathrm{mg} / \mathrm{dl}(5.6 \mathrm{mmol} / \mathrm{l})$.

-International Diabetes Federation (IDF) criteria [27] Central obesity (defined as waist circumference but can be assumed if BMI > $30 \mathrm{~kg} / \mathrm{m}^{2}$ ) with ethnicity-specific values, ${ }^{*}$ plus two of the following: (1) Triglycerides $150 \mathrm{mg} / \mathrm{dl}(1.7$ $\mathrm{mmol} / \mathrm{l})$ or greater ; (2) HDL-cholesterol $<40 \mathrm{mg} / \mathrm{dl}(1.03 \mathrm{mmol} / \mathrm{l})$ in men and < $50 \mathrm{mg} / \mathrm{dl}(1.29 \mathrm{mmol} / \mathrm{l})$ in women ; (3) BP 130/85 mmHg or greater (4) Fasting glucose $100 \mathrm{mg} / \mathrm{dl}(5.6 \mathrm{mmol} / \mathrm{l})$ or greater.

*To meet the criteria, waist circumference must be: for Europeans, $>94 \mathrm{~cm}$ in men and $>80 \mathrm{~cm}$ in women; and for South Asians, Chinese, and Japanese, $>90 \mathrm{~cm}$ in men and $>80 \mathrm{~cm}$ in women. For ethnic South and Central Americans, South Asian data are used, and for sub-Saharan Africans and Eastern Mediterranean and Middle East (Arab) populations, European data are used.

\subsection{Statistical methods}

SPSS (version 20.0) was used for all statistical analyses. Student's $t$-tests and analysis of variance (ANOVA) were used to evaluate differences in mean for study groups. Continuous variables were expressed as mean \pm standard deviation. Chi square was used to evaluate the difference in mean categorical variables. The prevalence estimates for MetS according to the three definitions were determined separately (WHO, IDF, NCEP-ATPIII). Using previously described methods, $95 \%$ confidence intervals for prevalence estimates were determined [28]. All reported $P$ values are two tailed, and statistical significance was set at 0.05 levels.

\section{Anthropometric and biochemical findings}

\section{RESULTS}

The study consisted of 80 participants (28 males [35\%] and 52 [65\%] females) with mean age 24.4 \pm 5.87 years old. The mean age for the male was $24.96 \pm 6.76$ years, while the mean age for the female was $24.21 \pm 5.39$ years. Table 1 shows the baseline and clinical characteristics of the study population. Approximately, a quarter of male students $(28.6 \%)$ and female students $(25.0 \%)$ were overweight (BMI 25.0-29.9 kg/m²). Females were more likely to be obese (25.0\%) compared to males (14\%). The mean systolic blood pressure (sBP) and diastolic blood pressure (dBP) were higher for male students $(128.67 \pm 14.5 \mathrm{mmHg}$ and $79.64 \pm 11.9 \mathrm{mmHg}$ respectively) than for female students $(117.19 \pm 13.25 \mathrm{mmHg}$ and $73.52 \pm 10.50 \mathrm{mmHg}$ respectively). Male students also had a slightly higher mean WHR $(0.84 \pm 0.04)$ compared with the females $(0.81 \pm 0.06)$ while waist circumference and fasting serum glucose concentrations were similar for both groups respectively. However, mean serum HDL-C concentrations were higher among female students (1.72 \pm 0.59$)$ as compared with male students $(1.46 \pm 0.48)$. The mean serum triglyceride concentrations were higher, among males $(0.98 \pm 0.81)$ as compared with females $(0.70 \pm 0.33)$. It was observed that there was a significant difference between sexes for the following variables: $\operatorname{sBP}(P=0.000), \mathrm{dBP}(P=0.040), \operatorname{HDL}-\mathrm{C}(P=0.036)$ and WHR $(P=0.003)$. 
Table 1: Anthropometric and biochemical characteristics of participants and differences by gender

\begin{tabular}{|c|c|c|c|c|}
\hline \multirow{3}{*}{ Characteristics } & \multicolumn{3}{|c|}{ Gender } & \multirow{3}{*}{$P$-value ${ }^{a}$} \\
\hline & Total $(n=80)$ & Male students $(n=28)$ & Female students $(n=52)$ & \\
\hline & $(\%)$ & $(\%)$ & $(\%)$ & \\
\hline \multicolumn{5}{|l|}{ BMI $\left(\mathrm{kg} / \mathrm{m}^{2}\right)$} \\
\hline Underweight $(<18.5)$ & 1.3 & & 1.9 & $0.000^{\mathrm{b}}$ \\
\hline Normal (18.5-24.9) & 26.3 & 57.1 & 48.1 & \\
\hline Overweight (25.0-29.9) & 51.3 & 28.6 & 25.0 & \\
\hline \multirow[t]{2}{*}{ Obese $(\geq 30.0)$} & 21.3 & 14.3 & 25.0 & \\
\hline & Mean (SD) & Mean (SD) & Mean (SD) & \\
\hline Age & $24.48 \pm 5.87$ & $24.96 \pm 6.76$ & $24.21 \pm 5.39$ & 0.588 \\
\hline Glucose (mmol/l) & $4.13 \pm 0.63$ & $4.15 \pm 0.75$ & $4.12 \pm 0.56$ & 0.827 \\
\hline Systolic blood pressure (mmHg) & $121.21 \pm 13.90$ & $128.67 \pm 14.45$ & $117.19 \pm 11.89$ & 0.000 \\
\hline Diastolic blood pressure (mmHg) & $75.66 \pm 11.83$ & $79.64 \pm 13.25$ & $73.52 \pm 10.50$ & 0.040 \\
\hline Triglycerides (mmol/l) & $0.80 \pm 0.56$ & $0.98 \pm 0.81$ & $0.70 \pm 0.33$ & 0.091 \\
\hline High-density lipoprotein (mmol/l) & $1.63 \pm 0.57$ & $1.46 \pm 0.48$ & $1.72 \pm 0.59$ & 0.036 \\
\hline Waist circumference $(\mathrm{cm})$ & $79.94 \pm 11.24$ & $79.89 \pm 9.88$ & $79.96 \pm 12.00$ & 0.978 \\
\hline Waist-Hip Ratio (WHR) & $0.82 \pm 0.06$ & $0.84 \pm 0.04$ & $0.81 \pm 0.06$ & 0.003 \\
\hline MetS by ATPIII criteria & $1.06 \pm 0.24$ & $1.07 \pm 0.26$ & $1.06 \pm 0.24$ & $0.809^{\mathrm{c}}$ \\
\hline MetS by WHO criteria & $1.03 \pm 0.16$ & $1.07 \pm 0.26$ & $1.00 \pm 0.00$ & $0.051^{\mathrm{c}}$ \\
\hline MetS by IDF criteria & $1.06 \pm 0.27$ & $1.10 \pm 0.32$ & $1.06 \pm .0 .24$ & $0.423^{\mathrm{c}}$ \\
\hline \multicolumn{5}{|c|}{ 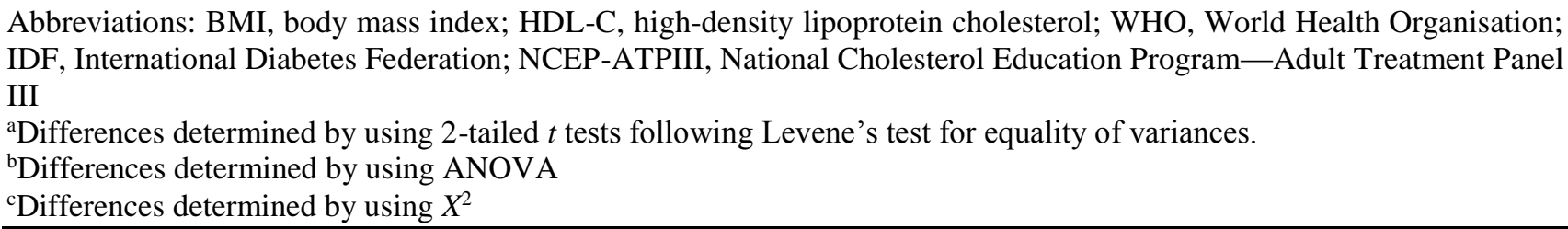 } \\
\hline
\end{tabular}

Table 2: Prevalence of metabolic syndrome components by gender according to NCEP-ATP III, WHO and IDF criteria.

\begin{tabular}{lccccccc}
\hline \multirow{2}{*}{ Gender } & \multicolumn{2}{l}{ NCEP-ATPIII } & \multicolumn{2}{c}{ WHO } & & \multicolumn{2}{c}{ IDF } \\
\cline { 2 - 7 } & Male & Female & Male & Female & Male & Female \\
\hline$N$ & 28 & 52 & 28 & 52 & 28 & 52
\end{tabular}

MetS criteria:

\begin{tabular}{|c|c|c|c|c|c|}
\hline Hypertension, $n(\%)$ & 7.1 & 5.8 & 3.6 & 10.7 & 5.8 \\
\hline Hyperglycaemia, $n(\%)$ & & & 7.1 & 3.6 & \\
\hline Low HDL cholesterol, $n(\%)$ & 7.1 & 1.9 & & 7.1 & 1.9 \\
\hline Hypertriglyceridaemia, $n(\%)$ & 3.6 & 3.8 & & 3.6 & 3.8 \\
\hline Body mass index $>30 \mathrm{~kg} / \mathrm{m}^{2}, n(\%)$ & & & 3.6 & 7.1 & 9.6 \\
\hline Waist circumference, $n(\%)$ & 7.1 & 5.8 & & 10.7 & 5.8 \\
\hline to-Hip ratio, $n(\%)$ & & & 7.1 & & \\
\hline
\end{tabular}

WHO, World Health Organization; IDF, International Diabetes Federation;

NCEP-ATPIII, National Cholesterol Education Program-Adult Treatment Panel III 
Table 2 shows the prevalence of MetS components by gender according to NCEP-ATP III, WHO and IDF criteria. The most common MetS components among female students using the NCEP-ATP III criteria were high blood pressure $(5.8 \%)$ and abdominal obesity $(5.8 \%)$, whilst reduced HDL-C concentrations, high blood pressure and abdominal obesity were most common among male students (at $7.1 \%$ for each component mentioned). According to WHO, abdominal obesity $(7.1 \%)$ and hyperglycaemia $(7.1 \%)$ were the most common MetS components among male students. The most common MetS components among participants using the IDF criteria were abdominal obesity and high blood pressure. While some participants did not meet the MetS criteria of the NCEP-ATP III, WHO and IDF criteria (50\%), manyhad one $(30 \%)$ or two $(13.8 \%)$ components and may be at risk of developing the syndrome in the future.

Table 3: Prevalence of MetS according to three definitions (NCEP-ATPIII, WHO and IDF)

\begin{tabular}{|c|c|c|c|c|c|c|}
\hline \multirow[b]{2}{*}{$N$} & \multicolumn{2}{|c|}{ NCEP-ATPIII } & \multicolumn{2}{|c|}{ WHO } & \multicolumn{2}{|c|}{ IDF } \\
\hline & \multicolumn{2}{|c|}{80} & \multicolumn{2}{|c|}{80} & \multicolumn{2}{|c|}{80} \\
\hline MetS Yes: & \multicolumn{2}{|c|}{5} & \multicolumn{2}{|c|}{2} & \multicolumn{2}{|c|}{6} \\
\hline MetS No: & \multicolumn{2}{|c|}{75} & \multicolumn{2}{|c|}{78} & \multicolumn{2}{|c|}{74} \\
\hline Percentage, $n(\%)$ & \multicolumn{2}{|c|}{6.3} & \multicolumn{2}{|c|}{2.5} & \multicolumn{2}{|c|}{7.5} \\
\hline Gender & Male & Female & Male & Female & Male & Female \\
\hline$N$ & 28 & 52 & 28 & 52 & 28 & 52 \\
\hline MetS Yes: & 2 & 3 & 2 & 0 & 3 & 3 \\
\hline MetS No: & 26 & 49 & 26 & 52 & 25 & 48 \\
\hline Percentage, $n(\%)$ & 7.1 & 5.8 & 7.1 & 0 & 10.7 & 5.8 \\
\hline
\end{tabular}

WHO, World Health Organisation; IDF, International Diabetes Federation; NCEP-ATPIII, National Cholesterol Education Program-Adult Treatment Panel III

Table 3 shows the prevalence of MetS as defined using the NCEP-ATP III, WHO and IDF diagnostic criteria. Using the NCEP-ATP III definition, the prevalence of MetS was $6.3 \%$ overall (7.1\% among male students and 5.8\% among female students) in the study population, $2.5 \%$ according to WHO definition $(7.1 \%$ among male students) and application of the IDF definition in this study population yielded a MetS prevalence of $7.5 \%$ overall (10.7\% of male students and $5.8 \%$ of female students).

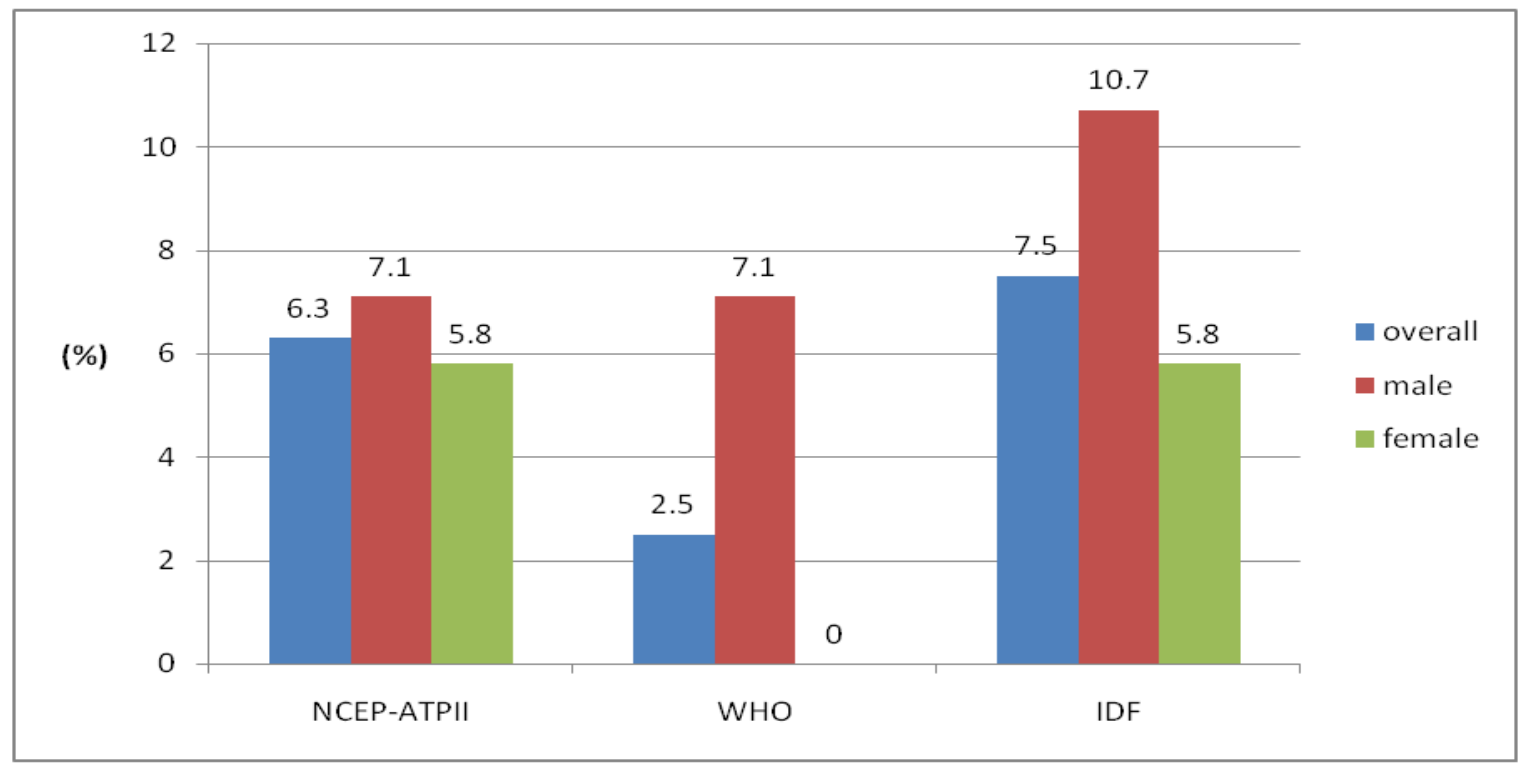

Figure 1: Prevalence of MetS according to three definitions (NCEP-ATPIII, WHO and IDF) 
The anthropometric and biochemical Characteristics of participants fulfilling the three definitions (NCEP-ATPIII, WHO, IDF) of MetS are shown in Table 1. All risk factors were almost significantly different $(P<0.05)$ when comparing those with and without MetS. Young adults who were classified as having MetS had a significantly higher BMI, waist circumference, triglycerides, systolic blood pressure and diastolic blood pressure, and lower HDL-C than those who were negative for MetS.

\section{DISCUSSION}

Findings from this study confirm a low prevalence of MetS among students of FHST in EBSU, Ebonyi State, Nigeria. Using the NCEP-ATP III definition, the prevalence of MetS was found to be 6.3\%, using WHO definition it was $2.5 \%$ while the IDF criteria yielded an even higher prevalence of 7.5\% (Figure 1). Results from the study suggest that male students were at greater risk for MetS (NCEP-ATP III 7.1\%, WHO 7.1 and IDF 10.5\%) than female students (NCEPATP III 5.8\% and IDF 5.8\%). These prevalence rates are lower than 20-25\% estimated for adults population globally by IDF [29]; 28.1\% reported using IDF criteria in a systematic review of published studies in adult Nigerians [30] and 35\% reported among healthy elderly South-western Nigerians. [31] The prevalence of MetS increased markedly with age and was most prevalent among participants with BMI $\geq 30 \mathrm{~kg} / \mathrm{m}^{2}$ (male, $14.3 \%$ and females, $25 \%$ ). This is attributed to the fact that ageing is associated with emergence of insulin resistance, diversion of fat from peripheral to the central sites with progressive redistribution of the fat stores more to the intra-abdominal visceral region. [31] In addition, neurohormonal alterations associated with ageing can predispose, promote and enhance mechanistic pathogenesis of MetS. [32]

Lastly, the number of participants with one (30\%) or two (13.8\%) MetS components suggest that certain individuals are potentially at risk of developing the syndrome. Little information exists concerning the prevalence and epidemiological characteristics of MetS in sub-Sahara Africa especially in young adults. The first reported MetS study in the region was conducted in the mid-90s in Cameroon [33] which found a 1.5\% and 1.3\% prevalence of MetS among urban dwelling women and men using IDF criteria [8]; however, the study did not measure HDL-C concentrations. A second study conducted in 2004 by [34] in Seychelles, found a high prevalence of MetS where 25\%-30\% of their study population had the syndrome. A community based study conducted in Tanzania in 2009 reported a 38\% prevalence of MetS although the study sample size was small [35]. In Nigeria, a study involving adults in semi-urban and rural communities found a prevalence of MetS to be 18\% [13], [36]in their study among rural Nigerians reported a 12.1\% prevalence of MetS, prevalence of $15.9 \%$ was reported in South eastern Nigeria [13] and 14.9\% was reported in Abuja, North central Nigeria. [14]

The prevalence estimates of the present study population are lower than what was reported in Cameroon, rural Nigeria, Ethiopia, Ghana $[8,12,36,37]$ Seychelles and Tanzania $[38,39]$. The study findings were lower than other reports of MetS prevalence of developed countries over the last few years including the USA [1], Portugal [40], Turkey [41], Saudi Arabia [9], and India. [10] Several other studies before now have also evaluated MetS prevalence using more than one definition including those established by NCEP-ATP III, WHO and IDF. [34,36].

Previous studies conducted in sub-Saharan Africa including in Benin and in south-western Nigeria have identified a high prevalence of cardiometabolic risk factors including abdominal obesity $(32.0 \%$ and $14.7 \%$, respectively) and HTN ( $23.0 \%$ and $28.0 \%$, respectively) $[42,43]$ whereas this present study identified $2.5 \%$ of the study population to be prediabetic and high prevalence of abdominal obesity (21.3\%), HTN (18.8\%), high TG (6.3\%) and reduced HDL-C $(23.8 \%)$. The Prevalence of $2.5 \%$ to $7.5 \%$ as seen in this study could be a reflection of the socioepidemiological characteristics of the study population (students) whom are young adults and still physically active. The current wave of epidemiological and nutritional transition that has occurred globally including in sub-Saharan African countries where lifestyle and behavioural changes, both products of modernization and urbanization, appears not to have taken place in the study population but preventative measures as well as interventional programs should be put in place as this epidemiological transition predispose to increased risk and incidence of CVD. [44]

The global prevalence of chronic non-communicable diseases (NCDs) is on the rise, with the majority of the growth occurring among populations in developing countries [45]. In sub-Saharan Africa, NCDs are projected to surpass infectious diseases by 2030 [46]. As young adult Nigerians adopt more western lifestyles, they also accumulate diseases associated with socio-technological and economic advancement such as metabolic syndrome and this carries great concern for safeguarding the health of the young adult Nigerians in general.

\section{CONCLUSION}

Prevalence of the MetS ranged from $2.5 \%$ to $7.5 \%$ and using the NCEP-ATP III definition, the prevalence of MetS was found to be $6.3 \%$, using WHO definition it was $2.5 \%$ while the IDF criteria yielded $7.5 \%$. Individually, some participants were identified as having MetS, but when the mean values of risk factors of the study population are taken into consideration, these participants, although obese, seem to be healthy. The number of participants with MetS may decrease once ethnic-specific cut-points have been developed.

The lipid profile of the study population were relatively normal since the mean TG $(0.80 \pm 0.56)$ and HDL-C $(1.63 \pm 0.57)$ of participants were within normal reference values whereas the fasting glucose levels of the study 
population were also normal since the mean glucose levels $(4.13 \pm 0.63)$ of participants were within normal reference values. However, $2.5 \%$ of the study population was found to be pre-diabetic and the proportion with high TG (6.3\%) and reduced HDL-C $(23.8 \%)$ appears to be high.

The WHR and WC of the study population were normal since mean WHR $(0.82 \pm 0.06)$ and WC $(79.93 \pm 11.24)$ which were the anthropometric indices used to determine abdominal obesity was below the cut-off for required detecting abdominal obesity but mean BMI $(25.67 \pm 4.65)$ of the participants showed that majority of the study population was overweight. The sBP and dBP were also normal since the mean sBP $(121.21 \pm 13.90)$ and $\mathrm{dBP}(75.66 \pm 11.83)$ were below the cut-off for MetS and also relatively normal. However, $18.8 \%$ were hypertensive and $21.3 \%$ abdominal obesity. The high prevalence of abdominal obesity supports the reasoning that it is an early step in the development of MetS and most of those diagnosed of MetS also had this risk factor. Individuals with and/or at risk of MetS ought to focus on weight management and engage in appropriate physical activity and energy consuming exercises.

The mean values of the risk factors (any three of abdominal obesity, hyperglycaemia, HTN, high TG and low HDL-C) used as criteria for the diagnosis of MetS were relatively normal in the study population thereby masking the presence of MetS, thus showing that the prevalence of MetS may gradually increase undetected unless individual members of the study population are subjected to laboratory investigation using different criteria for diagnosis of MetS. Therefore, traditional risk factors (abdominal obesity, hyperglycaemia, HTN, high TG and low HDL-C) might be late markers in diagnosis of MetS since findings from this study showed that MetS was only detected in participants that already had it. Moreover, future interventions by health policy makers and public health officials ought to focus on the individuals at risk for MetS who have one or two risk factors in order to control any potential burden of the syndrome.

\section{REFERENCES}

1. Alberti, K.G., Eckel, R.H., and Grundy, S.M. (2009). Harmonizing the metabolic syndrome: a joint interim statement of the International Diabetes Federation Task Force onEpidemiology and Prevention; National Heart, Lung, and Blood Institute; American Heart Association;World Heart Federation; International Atherosclerosis Society; and International Association for the Study of Obesity. Circulation,120:1640-1645.

2. Alberti, K.G., Zimmet, P., and Shaw, J. (2006). Metabolic syndrome-a new world-wide definition.A consensus statement from the International Diabetes Federation. DiabetesMedicine, 23:469-480.

3. Tokin A. (2004). The metabolic syndrome: A growing problem. European Heart Journal, Supplement 6: A32A42.

4. Meigs, J.B., Larson, M.G., and D'Agostino, R.B. (2002). Coronary artery calcification in type 2 diabetes and insulin resistance. The Famingham Offspring Study. Diabetes Care,25:1313-1319.

5. Ford, E.S., Giles, W.H., and Dietz, W.H. (2002). Prevalence of the metabolic syndrome among US adults: Findings from the Third National Health and Nutrition Examination. Survey.Journal of American Medical Association, 287:356-359.

6. Ford, E.S., Li, C., and Sattar, N. (2008). Metabolic syndrome and incident diabetes: current state of the evidence. Diabetes Care,31:1898-1904.

7. Miranda, P.J., Defronzo, R.A., Califf, R.M., and Guyton, J.R. (2005). Metabolic syndrome: definition, pathophysiology and mechanisms. American Heart Journal,149: 33- 45.

8. Fezeu, L., Balkau, B., Kengne, A.P., Sobngwi, E., and Mbanya, J.C. (2007). Metabolic syndromein a subSaharan African setting: central obesity may be the key determinant. Atherosclerosis, 193:70-76.

9. Al-Nozha, M., Al-Khadra, A., Arafah, M.R., Al-Maatouq, M.A., Khalil, M.Z., and Khan, N.B. (2005). Metabolic syndrome in Saudi Arabia. Saudi Medical Journal, 26:1918-1925.

10. Pemminati, S., Prabha, A.M.R., Pathak, R., and Pai, M.R. (2010). Prevalence of metabolic syndrome (MetS) using IDF 2005 guidelines in a semi urban south Indian (Boloor Diabetes Study) population of Mangalore. Journal of Associate Physicians India, 58:674-677.

11. Tran, A., Gelaye, B., Girma, B., Lemma, S., Berhane, Y., and Bekele, T. (2011). Prevalence of metabolic syndrome among working adults in Ethiopia. International Journal of Hypertension.

12. Gyakobo, M., Amoah, A.G.B., Martey-Marbell, D., and Snow, R.C. (2012). Prevalence of the metabolic syndrome in a rural population in Ghana. BMC Endocrine Disorders, 12:25.

13. Ulasi, I.I., Ijoma, C.K., and Onodugo, O.D. (2010). A community-based study of hypertension and car diometabolic syndrome in semi-urban and rural communities in Nigeria. BMC Health ServiceResources10:71.

14. Adediran, O., Akintunde, A.A., Edo, A.E., Opadijo, O.G., and Araoye, A.M. (2012). Impact of urbanization and gender on frequency of metabolic syndrome among native Abuja settlers in Nigeria. Journal of Cardiovascular Disease Resources, 3:191-196. 
15. Alebiosu, Co., and Odusan, B.O. (2004). Metabolic Syndrome in subjects with type 2 diabetes mellitus. Journal of National Medical Association, 96:817-821.

16. Isezuo, S.A. (2005a). Is high density lipoprotein cholesterol useful in diagnosis of metabolic syndrome in native Africans with type 2 diabetes? Ethnic Disorders, 15:6-10.

17. Reaven, G.M. (1988). Banting lecture: Role of insulin resistance in human disease. Diabetes, 37:1595-1607.

18. Marchesini, G., Melchionda, N., Apolone, G., Cuzzolaro, M., Mannucci, E., and Grossi, E.(2004).The metabolic syndrome in treatment seeking obese persons. Metabolism, 53:435-440.

19. Ismail, M.F. (2012). Metabolic syndrome among obese Qataris attending primary health care centers in Doha. 2010. Journal of Family and CommunityMedicine, 19:7-11.

20. Surana, S.P., Shah, D.B., Gala, K., Hoskote, S.S., Gill, N., and Joshi, A.R. (2008). Prevalence of metabolic syndrome in an Indian population using the NCEP ATP III. Journal of Association of Physicians India,56:865868.

21. Akintunde, A.A., Ayodele, O.E., Akinwusi, P.O., and Opadijo, G.O. (2011). Metabolic syndrome: Comparison of occurrence using three definitions in hypertensive patients. Clinical Medicine Resources, 9:26-31.

22. Termizy, H.M., and Matauzy, M. (2009). Metabolic syndrome and its characteristics among obese patients attending an obesity clinic. Singapore Medical Journal 50: 390-394.

23. Mottillo, S., Filion, K.B., Genest, J., Joseph, L., Pilote, L., Poirier, P., Rinfret, S.,and Schiffrin, E.L., and Eisenberg, M.J. (2010). The metabolic syndrome and cardiovascular risk a systematic review and metaanalysis. Journal of American College of Cardiology,56:1113-1132.

24. Hanson, R.L., Imperatore, G., Bennett, P.H., and Knowler, W.C. (2002). Components of the metabolic syndrome" and incidence of type 2 diabetes. Diabetes, 51:3120-3127.

25. World Health Organization [WHO]. (2008). Prevention of chronic disease: A vital investment. Geneva, Switzerland: Author.

26. Alberti, K.G., and Zimmet, P.Z. (1998). Definition, diagnosis and classification of diabetes mellitus and its complications. Part 1: diagnosis and classification of diabetes mellitus provisional report of a WHO consultation. Diabetic Medicine, 15:539-553.

27. Alberti, K.G., Zimmet, P.Z., and Shaw, J. (2005). The metabolic syndrome-a new worldwide definition. Lancet,366:1059-1062.

28. Chobanian, A.V., Bakris, G.L., and Black H.R. (2003). Seventh report of the Joint National Committee on Prevention, Detection, Evaluation, and Treatment of High Blood Pressure. Hypertension, 42(6):1206-1252

29. George, A., Paul, Z.M., and Jonath, S. (2006.) The International Diabetes Federation consensus worldwide definition of metabolic syndrome. International Diabetes Federation

30. Oguoma, V.M., Nwose, E.U., and Richards, R.S. (2015). Prevalence of cardio-metabolic syndrome in Nigeria: A systematic review. Public Health, 129:413-423.

31. Iloh, G.P., Ikwudinma, A.O., and Obiegbu, N.P. (2013). Obesity and its cardio-metabolic co-morbidities among adult Nigerians in a primary care clinic of a tertiary hospital in South-Eastern Nigeria. Journal of Family Medicine and Primary Care,2:20-26

32. Alshehri, A.M. Metabolic syndrome and cardiovascular risk. (2010). Journal of Family Community Medicine, 17:73-8

33. Motala, A.A., Mbanya, J.C., and Ramaiya, K.L. (2009). Metabolic syndrome in sub-Saharan Africa. Ethnicity \& disease, 19(2):S2-S8.

34. Kelliny, C., William, J., Riesen, W., Paccaud, F., and Bovet, P. (2008). Metabolic syndrome according to different definitions in a rapidly developing country of the African region. CardiovascularDiabetology, 7(27).

35. 28.Njelekela, M. A., Mpembeni, R., and Muhihi, A. (2009). Genderrelated differences in the prevalence of cardiovascular disease risk factors and their correlates in urban Tanzania. BMC Cardiovascular Disorders, 9(30).

36. Adegoke, O. A., Adedoyin, R. A., Balogun, M. O., Adebayo, R. A., Bisiriyu, L. A., and Salawu, A. A. (2010). Prevalence of metabolic syndrome in a rural community in Nigeria Metabolic Syndrome and Related Disorders, 8(1):59-62.

37. Zhang, J., Pronyuk, K.H., Kuliesh, O.V., and Chenghe, S. (2015). Adiponectin, Resistin and Leptin: Possible Markers of Metabolic Syndrome. Endocrinology and metabolic syndrome, 4:212.

38. Conti, E., Carrozza, C., Capoluongo, E. (2004). Insulin-like growth factor-1 as a vascular protective factor. Circulation, 110(15):2260-2265.

39. Deen, D. (2004). Metabolic syndrome: time for action. American Family Physician. 69:2875-2882.

40. Santos, A. C., and Barros, H. (2007). Impact of metabolic syndrome definitions on prevalence estimates: a study in a Portuguese community.Diabetes and Vascular Disease Research,4(4):320-327.

41. Can, A. S., and Bersot, T. P. (2007). Analysis of agreement among definitions of metabolic syndrome in nondiabetic Turkish adults: a methodological study.BMC Public Health, 7(353) 
42. Oladapo, O. O., Salako, L., Sodiq, O., Shoyinka, K., Adedapo, K., and Falase, A. O. (2010). A prevalence of cardiometabolic risk factors among a rural Yoruba south-western Nigerian population: a population-based survey.Cardiovascular Journal of Africa, 21(1):31.

43. Sodjinou, R., Agueh, V., Fayomi, B., and Delisle, H. (2008). Obesity and cardio-metabolic risk factors in urban adults of Benin: relationship with socio-economic status, urbanisation, and lifestylepatterns. BMC Public Health, 8(84).

44. Onyegbutulem, H. C., H-Onyegbutulem, P. I., Reimann, M., Li, J., Bornstein, S. R., and Schwarz, P. E. (2009). Metabolic syndrome in Africa: an emerging perspective. Hormone and Metabolic Research, 41(2)75-78.

45. Murray, C. J. L., and Lopez, A. D. (1997). Alternative projections of mortality and disability by cause 19902020: Global Burden of Disease Study. Lancet, 349 (9064):1498-1504.

46. Mathers, C.D., and Loncar, D. (2006). "Projections of global mortality and burden of disease from 2002 to 2030." PLoS Medicine, 3(11): article e442. 